\title{
The Convergence of the Codex Book and the e-Book
}

\author{
Robert K. Logan \\ Ontario College of Art and Design, Toronto, Canada; logan@physics.utoronto.ca
}

\begin{abstract}
We are proposing a platform for a new form of the book, the sBook, which is a combination of the codex book and the e-book. The sBook combines the advantages of these two formats of the book as well as some additional features that we have designed for this new hybrid book. We describe one possible form of an sBook, which is a codex book that has been "smart tagged" so that the book directs one's Enabler to a Web site that contains the digital form of the text of the codex book. The Enabler could be a desktop or notebook computer, a PDA or a smart phone such as the iPhone. As a result the sBook system - consisting of the codex book, the Enabler, and the Web site - is readable, searchable, networkable, updatable, smart and promotes "active reading". The book is very readable because the sBook still retains the codex format of ink on paper. Because the "smart tag" directs the reader to a Web site with the digital text and room for comments by readers and updates by the author, the sBook is searchable, networkable and updatable. Finally by incorporating a recommender system installed on the Enabler the sBook can match its content to that of the reader's research and information interests. The recommender system can also search the Net for pertinent information. This paper explores some of the possible applications of the sBook and its impact on authors, readers, publishers, booksellers and libraries.
\end{abstract}

Keywords: book, sBook, smart, "active reading", booksellers, publishers, libraries, searchable

Acknowledgement: The sBook was first conceived at the Strategic Innovation Lab (formerly the Beal Institute of Strategic Creativity) at the Ontario College of Art and Design in 2005 and is headed by the co-PIs RKL and Greg Van Alstyne. We wish to acknowledge the support of the Lab's director, Lenore Richards and the contributions that have been made to developing the SmartBook idea by the project team consisting of James Caldwell, Dave Gray, Peter Jones, Kathy Kawasaki, Gale Moore, Matt Ratto, Ramon Sangüesa, and Carlos Scolari.

\section{Starting Idea}

A group of researchers at the Strategic Innovation Lab at the Ontario College or Art and Design headed by Greg Van Alstyne and Robert K. Logan are proposing a new format or platform for books that combines the best features of a codex book and an e-book. One form of the sBook we are considering would use a smart tag, which could be a bar code, a QR code or an RFID tag depending on the application, with a standard printed codex or folio book that points one's Enabler to a Web site that has the text of the book in a digital format. The Enabler could be a desktop or notebook computer, a PDA or a smart phone such as the iPhone. The resulting hybrid book, which we call an sBook, combines the features and advantages of a codex book and an e-book as well as the added feature of a recommender system that is to be embedded in the Enabler. As a consequence the sBook is readable, searchable, networkable, smart and promotes "active reading".

The sBook is readable because of its codex format and the fact that ink on paper is the best way to read text. It is searchable because the smart tag points to a digital form of the book's content on a Web site. It is networkable because the Web site containing the digital form of the book is to be used for 
readers to share ideas about the book with each other and the author as is done on a blog. The author can comment on the readers' remarks and also update her book either on the basis of readers' comments or because of new developments in the field of study that the book addresses. Finally, the book is smart because it knows what the reader wants to know and it can recommend what parts of the book are of particular interest to the reader through the recommender system embedded in the Enabler. The recommender system has a profile of the reader's research needs and information interests and has an internal and an external component. The internal recommender system is able to highlight those portions of the book that will be of particular interest to the reader. The external recommender system would be largely Web based and would bring information external to the book to the attention of the reader. The Enabler and the Web site with the digital text would also have tools to facilitate active reading consisting of the following activities: "annotating, quoting, comparing, searching, taking notes, sharing" as described in detail by Gene Golovchinsky (www.fxpal.com/ publications/FXPAL-PR-08-468.pdf) in his article Reading in The Office (see also www.zenguide.co.uk/2008/05/going-shelfless/ for more on active reading.

The sBook system also allows a codex book to incorporate the advantages of hypertext through the Web interface. One would read the codex book with one' Enabler close at hand. The author could indicate places in the codex text where one could jump to another part of the book or to another source of information on the Web, which the reader of the codex form of the book could access with their Enabler through the Web and thereby enrich their reading experience. The text that will be hypertexted in the digital version of the book can be indicated in the printed book by using a special font to indicate where the hypertext link can be found in the digital version of the book.

Our proposal is not the first example of a system that combines a codex book with a digital form of the text. There are for example many textbooks that come with a CD-ROM containing the digital version of the book. Amazon has an Upgrade feature which allows a reader to access an electronic version of the book they order on the Internet for a selected number of books. It also allows the reader to add highlights, bookmarks, notes or tags to any page in the book. Norton publishers have a series of textbooks that they offer in a standard codex form with access to a digital version of the book and an electronic workbook (SmartWork). They also offer the ebook version of their textbook for approximately half the price of the codex version of the book. O'Reilly Media offers a number of their books in a print version, an ebook version and a bundled package of the two formats. Their e-book version comes in three digital formats, PDF, ePub and Mobipocket (for Kindle users).

The SmartBook system that we have just described should be regarded as SmartBook 1.0 and is presented as a scenario to help the reader imagine how some combination of a codex book and an e-book could create a reading system that has the best of both platforms. We are still in the midst of our research to design a platform that is a hybrid of the codex book and the e-book that will satisfy the needs, desires, expectations and latent behaviours of $21^{\text {st }}$ century readers. This is a progress report of work in progress.

What makes our sBook proposal different is that it incorporates so many useful features with the potential to change:

- the operations of libraries,

- the operations of bookstores,

- the operations of publishers,

- the way in which books are kept up to date,

- the way in which the book can become the focus of a social network,

- the way active reading is pursued,

- the way knowledge is shared,

- the way elearning operates, and

- the way researchers can more efficiently find the information they need for their projects without reading the whole book.

Our research agenda is to consider all possible configurations of sBooks and match them with possible applications in the future that tap into the needs, desires, expectations, and latent behaviours of potential readers and users. In this paper we will report on our preliminary progress in carrying out this agenda and describe a publishing project that 
will test the networking and upgrading features of an sBook. Our objective is not to design a device but rather a platform. The description of the device, which uses a tag to link with a Web, is used to give the reader a notion of the kind of platform we have in mind. It is basically a scenario to help us envisage the kind of platform we would like to develop and what properties we would like that platform to possess.

\section{A New Option for Publishers}

The sBook represents a third option for book publishers in addition to the standard printed codex book (option 1), and the various digital formats of the book such as an e-book or a book on a CD-ROM (option 2). Options 1 and 2 have their unique advantages. The chief advantage of the printed codex or folio book is that it is the best form yet devised for readability. The codex format is also better suited for quickly browsing the book to get the feel for it especially if the book has a detailed table of contents and index and is written so that the contents of the book are summarized in the first few pages of the book. The advantage of the digital book, on the other hand, is that it is the format of choice for searching, researching and engaging in active reading. The e-book has the advantage that for a complex subject one can easily search the content of the book for topics of particular interest to the reader and thereby tie together related themes. This is particularly true if the e-book is written taking advantage of hypertext.

The sBook has all the advantages of both options 1 and 2 and in addition it can customize the content of the book for the specific use of the reader and it can create a forum for the discussion of the book. These features are particularly useful for books that are written and used for research. There is less of a need for the searchability of the sBook format for a novel or a book of poetry unless that book is a classic that is frequently studied by scholars and students. But even for books that are purely literary the ability of readers to network with each other and possibly the author could be a distinct advantage.

If the sBooks succeed in penetrating the market they will have an enormous impact of book publishing, booksellers, libraries and schools. Book publisher will not only have to print and distribute codex books they will also have to maintain a Web site for each book they publish.

As the number of sBooks increases there will be an impact on libraries. Imagine a library of sBooks in which a user enters with their recommender system on their Enabler and are directed to those volumes that are of most interest to them. Part of the function of the reference librarian will be taken over by the sBook. The library edition of the sBook will have to have a RFID tag that can transmit a radio signal over a long distance.

The sBooks will also impact bookstores. Imagine walking into a bookstore with one's Enabler with an embedded recommender system and being directed to the books one would want to buy. How convenient!

\section{A New Option for Authors}

Printed books have been limited to text and pictures (illustrations, photographs and reproduction of fine art). With the sBook platform a whole new world opens up because the ability to use hypertext links to the Web site associated with the book as described above to enrich the text and illustrations of the printed book. In addition to text and illustrations the author can now add video and audio files to enrich the traditional content of a printed book. When we first conceived the sBook project we thought that fictional sBooks would not be of much interest but as a result of a number of fiction authors we met at the BookCampTO held in Toronto June 6, 2009 we discovered there is an interest in creative writers to take advantage of the features of the sBook platform to create a new kind of art form, a novel or a short story that incorporates in the text pictorial elements, video clips and audio bites. We also found a publisher interested in publishing in this format. This is very exciting - the notion of creating a new art form on the sBook platform, a mashup of text, pictures, video and audio!! 


\section{Laws of the Media (LOM)}

To gain a deeper insight into the nature of the codex book and the sBook that we are proposing let us apply McLuhan's (McLuhan 1975 \& McLuhan and McLuhan 1988) Laws of the Media (LOM) for these two media.

LOM consist of the following four laws:

1. Every medium or technology enhances some human function.

2. In doing so, it obsolesces some former medium or technology, which was used to achieve the function earlier.

3. In achieving its function, the new medium or technology retrieves some older form from the past.

4. When pushed far enough, the new medium or technology reverses or flips into a complementary form.

\subsection{LOM Codex Book}

Enhances: the storage of and access to information

Obsolesces: oral tradition or myths

Retrieves: memory

Flips into: e-book

\subsection{LOM sBook}

Enhances: the codex book and hence readability, searchability, relevance, currency, active reading

Obsolesces: the traditional library and the manual search

Retrieves: the reference librarian and the book club

Flips into: the smart library and the online symposium

\section{Protecting Copyright}

In order to fulfill all the functions of the sBook that we have identified such as their use in a library or their availability at a bookstore and still protect the copyright of the authors the sBook will have to have three levels of access:
1. The privately owned version - The owner of the book can read the book in the traditional manner and also capture the data in the book electronically and transform it at will. They will be able to create a personal searchable library with a smart catalog from their collection of sBooks.

2. The library version - The library patrons will be able to read the book in hard copy and/or access the entire book electronically, which will allow them to search for and access items of interest in the sBook without having to read the entire book. This will facilitate a great deal of library research as scholars will be able to quickly access the material they need by organizing Google-like searches. They will be restricted, however, from the wholesale copying of the contents of the book onto their SB Reader or any third medium. A certain number of lines of text for the purpose of quoting will be allowed depending on the discretion of the publisher and the author.

3. The bookstore version - While the book sits in a bookstore before it is purchased it will be searchable but not copy-able. A collection of sBooks in the bookstore will allow customers to find a book or books that meet their interest and describe to them where in the store they may find them. They will be able to access the book electronically but they will not be able to copy any of the pages of the book except promotional pages at the discretion of the publisher and the author.

\section{5. sBook Applications}

In this section we describe a number of potential uses or application that can be made with the sBook system.

\subsection{Other media}

We begin by noting that the use of the sBook platform is not limited to books as one might wish to consider smart journals, smart magazines, and smart newspapers. Some features of a smart journal already exist as some journals are published in both a print and a digital form with space reserved in the digital form for reader comments. Most online versions of newspapers have a mechanism for reader comments but unfortunately they 
are too often used for flaming or promoting a particular political point of view rather than being used for entering into constructive serious dialogues.

\subsection{Print-on-demand}

A number of out of print book have been scanned by the Gutenberg Project, the Million Books Project and Google. It would be a real service to scholarship if some of these books could be offered in a bundle of the digital version and the print version produced by a print-on-demand (POD) service. Publishers might also wish to make their out of print books available in a digital/POD bundle.

Smart libraries - Imagine students and research faculty using their Enabler at their library's information commons to find the research material for their various projects.

\section{A Partial Prototype}

Two of the features of the sBook will be tested when my book Understanding New Media: Extending Marshall McLuhan, an update of McLuhan's Understanding Media: Extensions of Man is released in the summer of 2009. A Web site will be created that readers of the book will be directed to so that they can leave comments, which I may choose to respond to in the spirit of a blog. I will also use this Web site to continually update my book.

\section{Does the sBook System Actually Require the Incorporation of the Printed Book?}

There is a division of opinion among members of our research team and those who have commented on our project as to whether or not the format of the printed book will ultimately survive. Many believe that the printed book will basically be eclipsed by the e-book and that it will be relegated to special uses, as is the case of the scroll, which plays a central role in the weekly, or thrice weekly reading of the Torah in synagogues throughout the world. They argue that the ebook is just as easy to read as a printed book and that it is an extremely convenient medium especially when stored on an e-book reader because one can access hundreds of books without having the problem of porting and storing them.

I personally am of the opinion that the printed book will survive because it is still the best medium for close and careful reading. When I asked a group of authors at BookCampTO whether they edit their wordprocessed texts on paper or on a screen all 25 who responded said they edited their final version on paper. The same was true a group of 17 academic writers at the Media Ecology Association conference. One writer, however, said that she found that she caught some errors on screen that she did not catch on paper and vice-versa. I believe the reason that writers favour paper for editing over the screen is that they can focus better with ink on paper rather than with pixels on a screen. The reason for this preference can be explained in terms of our bicameral brain. We use the left side of our brain for language activities like speaking, writing and reading and we use the right side of our brain for visual and musical activities. When one reads on a screen one first uses the right brain to integrate the pixels into a gestalt that is then read by the left-brain so there is a lot of traffic flowing through the corpus collosum. A reader reading ink on paper only needs to use their left brain and therefore is less distracted and hence more focused.

I do not pretend that all readers will be convinced by this argument but we can all agree that the printed codex book will be around for quite a long time. But more importantly as many have pointed out it is not always necessary for the printed book to be part of the sBook platform. One could well imagine a publisher publishing an e-book part using the features of the sBook platform to take advantage of the way the Web site becomes an extension of the book through its features of social networking, author updates, "active reading," and its capability of creating new forms of hybrid text that incorporate video or audio clips. Publishers of e-books could also add a feature to their e-book that allows the purchasers of the e-book to make use of a print-on-demand device to create a hard copy of the book should they so desire. 


\section{An Invitation}

To conduct our sBook research we have created a Google Group discussion group called Rethinking the Book. Readers of this article interested in joining our Rethinking the Book Google discussion group should email me at logan@physics.utoronto.ca and they will be included in the group. 